Journal of Advanced Research in Fluid Mechanics and Thermal Sciences

\title{
Radiation and Chemical Reaction Effects on MHD Convective Flow over a Porous Plate through a Porous Medium with Heat Generation
}

\author{
Bommanna Lavanya ${ }^{1, *}$ \\ Department of Mathematics, MIT MAHE, Manipal-576104, KA, India
}

\begin{tabular}{ll} 
ARTICLE INFO & ABSTRACT \\
\hline $\begin{array}{l}\text { Article history: } \\
\text { Received 27 August 2019 }\end{array}$ & $\begin{array}{l}\text { The present paper analyzes the radiation and chemical reaction effects on a steady } \\
\text { Received in revised form 20 October 2019 }\end{array}$ \\
$\begin{array}{l}\text { laminar forced convection flow of a viscous incompressible electrical conducting fluid } \\
\text { over a flat plate embedded in a porous medium in the presence of heat generation. } \\
\text { Available online 21 March 2020 }\end{array}$ & $\begin{array}{l}\text { The governing equations are transformed by using similarity transformation and } \\
\text { resultant dimensionless equations are solved numerically using the Runge-Kutta } \\
\text { method with Shooting technique. The effects of various governing parameters on } \\
\text { velocity, temperature, concentration, skin-friction coefficient, Nusselt number and } \\
\text { Sherwood number are shown in figures and tables and analyzed in details. }\end{array}$
\end{tabular}

Keywords:

\section{Introduction}

Combined heat and mass transfer in fluid-saturated porous media finds applications in a variety of engineering processes such as heat exchanger devices, petroleum reservoirs, chemical catalytic reactors and processes, geothermal and geophysical engineering, moisture migration in a fibrous insulation and nuclear waste disposal and others. Lai and Kulacki [1] discussed the coupled heat and mass transfer by mixed convection from a vertical plate in a saturated porous medium. Many problems of Darcian and non-Darcian mixed convection about a vertical plate had been reported, as in Hsu and Cheng [2], Vafai and Tien [3]. Bejan and Khair [4] investigated the free convection boundary layer flow in a porous medium owing to combined heat and mass transfer.

Magnetohydrodynamic(MHD) flows have applications in meteorology, solar physics, cosmic fluid dynamics, astrophysics, geophysics and in the motion of earth's core. In addition, from the technological point of view, MHD free convection flows have significant applications in the field of stellar and planetary magnetospheres, aeronautical plasma flows, chemical engineering and electronics. Raptis [5] studied mathematically the case of time varying two dimensional natural

\footnotetext{
*Corresponding author.

E-mail address: lavanya.b@manipal.edu (Bommanna Lavanya)
} 
convective flow of an incompressible, electrically conducting fluid along an infinite vertical porous plate embedded in a porous medium. Helmy [6] analyzed MHD unsteady free convection flow past a vertical plate embedded in a porous medium. Elabashbeshy [7] studied heat and mass transfer along a vertical plate in the presence of magnetic field. Chamkha and Khaled [8] investigated the problem of coupled heat and mass transfer by MHD free convection from an inclined plate in the presence of internal heat generation or absorption.

In the context of space technology and in the processes involving high temperature, the effects of radiation are vital importance. Recent developments in hypersonic flights, missile re-entry, rocket, rocket combustion chambers, power plants for inter planetary flight and gas cooled nuclear reactors, have focused attention on thermal with laminar free convection heat transfer from a vertical plate was investigate by Cess [9] for an absorbing, emitting fluid in the optically thick region, using the singular perturbation technique. Arpaci [10] considered a similar problem in both the optically thick regions and used the approximate integral technique and firstorder profiles to solve the energy equation. Raptis [11] analyzed the thermal radiation and free convection flow through a porous medium bounded by a vertical infinite porous plate by using a regular perturabation technique. Mohammed Ibrahim and Bhaskar Reddy [12] studied the effects of radiation and mass transfer effects on MHD free convection flow a long a stretching surface with viscous dissipation and heat generation. Damesh et al., [13] found the similarity analysis of magnetic field and radiation effects on forced convection flow.

The study of heat generation or absorption effects in moving fluids is important in the view of several physical problems, such as fluids undergoing exothermic or endothermic chemical reactions. Vajravelu and Hadjinicolaou [14] studied the heat transfer characteristics in the laminar boundary layer of a viscous fluid over a stretching sheet with viscous dissipation or frictional heating and internal heat generation. Hossain et al., [15] studied problem of the natural convection flow along a vertical wavy surface with uniform surface temperature in the presence of heat generation/absorption. Few other recent related researches can be found in [16-20].

However, the interaction of radiation and chemical reaction effects on steady MHD forced convection flow in a porous medium with heat generation has received little attention. Hence, the object of the present paper is to analyze the radiation and chemical reaction effects on steady MHD forced convection boundary layer flow past a semi-infinite moving vertical plate embedded in a porous medium by taking heat generation into account. The governing equations are transformed by using similarity transformation and resultant dimensionless equations are solved numerically using the Runge-Kutta method with Shooting technique. The effects of various governing parameters on the velocity, temperature, concentration, skin-friction coefficient, Nusselt number and Sherwood number are shown in figures and tables and analyzed in detail.

\section{Methodology}

A steady two-dimensional laminar forced convection flow of a viscous incompressible electrically conducting and radiating fluid over a flat plate of very small thickness and much larger breadth, embedded in a porous medium, in the presence of heat generation or absorption is considered. The flow is assumed to be in the $x$-direction, which is taken along the semi-infinite plate and $y$-axis normal to it. The plate is maintained at a constant temperature $T_{w}$, which is higher than the constant free stream temperature $T_{\infty}$ and a constant concentration $C_{w}$, which is greater than the constant concentration $C_{\infty}$ of the surrounding fluid. A uniform magnetic field is applied in the direction perpendicular to the plate. The fluid is assumed to be slightly conducting, and hence the magnetic Reynolds number is much less than unity and the induced magnetic field is negligible 
in comparison with the applied magnetic field. It is further assumed that there is no applied voltage, so that electric field is absent. In the analysis of the flow in porous media, the differential equations governing the fluid motion is based on Darcy's law, which accounts for the drag exerted by the porous media. Thermal radiation is included in the energy equation. The governing equations of such type of flow are (with the application of Darcy's law)

Continuity equation:

$$
\frac{\partial u}{\partial x}+\frac{\partial v}{\partial y}=0
$$

Momentum equation:

$$
u \frac{\partial u}{\partial x}+v \frac{\partial u}{\partial y}=v \frac{\partial^{2} u}{\partial y^{2}}+g \beta\left(T-T_{\infty}\right)+g \beta^{*}\left(C-C_{\infty}\right)+\frac{v}{K^{\prime}}\left(u-U_{\infty}\right)-\frac{\sigma B_{0}^{2}}{\rho} u
$$

Energy equation:

$$
u \frac{\partial T}{\partial x}+v \frac{\partial T}{\partial y}=\frac{k}{\rho c_{p}} \frac{\partial^{2} T}{\partial y^{2}}-\frac{1}{\rho c_{p}} \frac{\partial q_{r}}{\partial y}+\frac{Q_{0}}{\rho c_{p}}\left(T-T_{\infty}\right)
$$

Species equation:

$$
u \frac{\partial D}{\partial x}+v \frac{\partial D}{\partial y}=D \frac{\partial^{2} C}{\partial y^{2}}-K_{r}\left(T-T_{\infty}\right)
$$

Here $u$ and $v$ are the components of velocity respectively in the $x$ and $y$ directions. $T$ - the temperature, $\mu$ - the coefficient of fluid viscosity, $\mathrm{C}$ - the concentration, $\rho$ - the fluid density, $v=\frac{\mu}{\rho}$. the kinematic viscosity, $c_{p}$ - the specific heat at constant pressure, $\mathrm{g}$ - the acceleration due to gravity, $\beta$ - the coefficient of thermal expansion and $\beta^{*}$ - the coefficient of concentration expansion, $\mathrm{K}^{\prime}$ - the permeability of the porous medium, $k_{r}$ - the chemical reaction parameter, $U_{\infty}$ - the free stream velocity, $\sigma$ - electrical conductivity $B_{0}$ - the magnetic field intensity, $\mathrm{k}$ - the thermal conductivity of the fluid, $q_{r}$ - the radiative heat flux, D- the mass diffusivity.

Using Rosseland approximation for radiation (Brewster [21]) we can write

$$
q_{r}=-\frac{4 \sigma_{s}}{3 K_{e}} \frac{\partial T^{4}}{\partial y}
$$

where $\sigma_{s}$ is the Stefan-boltzman constant

$$
K_{e} \text { - The absorption coefficient }
$$

Assuming that the temperature difference within the flow is such that $T^{4}$ may be expanded in Taylor series and expanding $T^{4}$ about $T_{\infty}$ and neglecting higher orders.

we get $T^{4}=4 T_{\infty}^{3} T-3 T_{\infty}^{4}$

Therefore eq. (3) becomes

$$
u \frac{\partial T}{\partial x}+v \frac{\partial T}{\partial y}=\frac{k}{\rho c_{p}} \frac{\partial^{2} T}{\partial y^{2}}-\frac{16 \sigma T_{\infty}^{3}}{3 K^{\prime} \rho c_{p}} \frac{\partial q_{r}}{\partial y}+\frac{Q_{0}}{\rho c_{p}}\left(T-T_{\infty}\right)
$$

The appropriate boundary conditions for the velocity, temperature and concentration field are

$$
\begin{aligned}
& \mathrm{u}=0, \quad \mathrm{v}=0, \mathrm{~T}=T_{w}, \mathrm{C}=C_{w} \text { at } \mathrm{y}=0, \\
& \mathrm{u}=U_{\infty}, \mathrm{T}=T_{\infty}, \mathrm{C}=C_{\infty} \quad \text { as } \mathrm{y} \rightarrow \infty .
\end{aligned}
$$


Here $T_{w}$ is the wall temperature.

For the consideration of porous plate, embedded in a porous medium, a suction velocity is given through the plate. The boundary condition for the velocity component $\mathrm{v}$, then becomes $\mathrm{v}=v_{w}, v_{w}=$ $\frac{v_{0}}{\sqrt{x}}$ is the velocity of suction, $v_{0}$ is the initial value of the suction.

The eqs. (2) - (4) are coupled and nonlinear partial differential equations and hence analytical solution is not possible. Therefore, numerical technique is employed to obtain the required solution. Numerical computations are greatly facilitated by non-depersonalization of the equations. Proceeding with the analysis, we introduce the following similarity transformations and dimensionless variables which will convert the partial differential equations from two independent variables $(x, y)$ to a system of coupled, non-linear ordinary differential equation in a single variable (n) i.e., coordinate normal to the plate.

In order to write the governing equations and boundary conditions in dimensionless form, the following non-dimensional quantities are introduced.

$$
\begin{aligned}
& u=\frac{\partial \varphi}{\partial y}, \quad v=\frac{\partial \varphi}{\partial x}, G_{r}=\frac{g \beta\left(T_{w}-T_{\infty}\right) x}{U_{\infty}^{2}}, G_{c}=\frac{g \beta^{*}\left(C_{w}-C_{\infty}\right) x}{U_{\infty}^{2}}, \theta=\frac{T-T_{\infty}}{T_{w}-T_{\infty}}, \varphi=\frac{C-C_{\infty}}{C_{w}-C_{\infty}}, \\
& M=\frac{\sigma B_{0}^{2} x}{\rho U_{\infty}}, Q=\frac{Q_{0} x}{\rho C_{p} U_{\infty}}, K_{r}=\frac{2 K_{r}^{\prime} x}{U_{\infty}}, \eta=y \sqrt{\frac{U_{\infty}}{v x}}, \psi=\sqrt{U_{\infty} v x} f(\eta), S c=\frac{v}{D}, \operatorname{Pr}=\frac{\mu c_{p}}{k} \\
& R=\frac{k k^{*}}{4 \sigma T_{\infty}^{3}}, K=\frac{v x}{K U_{\infty}}
\end{aligned}
$$

where $\psi$ is the stream function, $\theta$ - the non-dimensional temperature function, $\phi$ - the nondimensional concentration, $\mathrm{Gr}$ - the thermal Grashof number, Gc - the solutal Grashof number, M the magnetic field parameter, $\mathrm{K}$ - the permeability parameter, $\mathrm{Pr}$ - the Prandtl number, $\mathrm{Sc}-$ the Schmidt number, $\mathrm{Q}$ - the heat generation or absorption coefficient , $\mathrm{Kr}$ - chemical reaction parameter.

The mass conservation equation ( 1 ) is satisfied by Cauchy-Riemann Equations

$$
u=\frac{\partial \varphi}{\partial y} \quad \text { and } v=\frac{\partial \varphi}{\partial x}
$$

In view of the Equation (8), the equations (2),(3) and (4) reduce to the following nondimensional form

$$
\begin{aligned}
& f^{\prime \prime \prime}+\frac{1}{2} f f^{\prime \prime}-K(f-1)+G r \theta+G c \varphi-M f^{\prime}=0 \\
& \frac{1}{p r}\left[1+\frac{4}{3 R}\right] \theta^{\prime \prime}+\frac{1}{2} f \theta^{\prime}+Q \theta=0 \\
& \varphi^{\prime \prime}+S c f \varphi^{\prime}-K r \varphi=0
\end{aligned}
$$

The corresponding boundary conditions are

$$
\begin{aligned}
& f^{\prime}=0, \quad f=S, \quad \theta=1, \quad \phi=1 \quad \text { at } \eta=0 \text {, } \\
& f^{\prime}=1, \quad \theta=0, \quad \phi=0 \quad \text { at } \eta \rightarrow \infty
\end{aligned}
$$

where $\mathrm{f}$ is the dimensionless stream function, $S=\frac{-2 v_{0}}{u_{\infty} v}$ is the dimensionless suction velocity and primes denote partial differentiation with respect to the variable $\eta$. The skin-friction coefficient, 
Nusselt number and Sherwood number are important physical parameters for this type of boundary layer flow. Knowing the velocity field, the skin-friction at the plate can be obtained, which in nondimensional form is given by

$$
C_{f}=2(\operatorname{Re})^{\frac{1}{2}} f^{\prime \prime}(0)
$$

Knowing the temperature field, the rate of heat transfer coefficient can be obtained, which in non-dimensional form, in terms of the Nusselt number, is given by

$$
N u=-(\operatorname{Re})^{\frac{1}{2}} \theta^{\prime}(0)
$$

Knowing the concentration field, the rate of mass transfer coefficient can be obtained, which in non-dimensional form, in terms of the Sherwood number, is given by

$$
S h=-(\operatorname{Re})^{\frac{1}{2}} \varphi^{\prime}(0)
$$

where $\operatorname{Re}=\frac{U_{\infty} x}{v}$ is the Reynolds number.

\section{Solution of the Problem}

The set of coupled non-linear governing boundary layer eqs. (9) - (11) together with the boundary conditions (12) are solved numerically by using Runge Kutta fourth order technique along with shooting method. First of all, higher order non-linear differential Eqs. (9) - (11) are converted into simultaneous linear differential equations of first order and they are further transformed into initial value problem by applying the shooting technique [22]. The resultant initial value problem is solved by employing Runge-Kutta fourth order technique. The step size $\Delta \eta=0.05$ is used to obtain the numerical solution with decimal place accuracy as the criterion of convergence. From the process of numerical computation, the skin friction coefficient, the Nusselt number and the Sherwood number, which are respectively proportional to $f^{\prime \prime}(0),-\theta^{\prime}(0)$ and $-\phi^{\prime}(0)$, are also sorted out and their numerical values are presented in a tabular form.

\section{Results and Discussion}

As a result of the numerical calculations, the dimensionless velocity, temperature and concentration distributions for the flow under consideration are obtained and their behaviour have been discussed for variations in the governing parameters viz., In the present study we adopted the following default parameter values $G r=3.0, G c=2.0, M=0.5, K=0.5, P r=0.71, \quad R=$ $0.5, Q=0.5, S c=0.6, S=1.0, \mathrm{Kr}=1.0$. All the graphs therefore correspond to these values unless specifically indicated on the appropriate graph.

The influence of the thermal Grashof number Gr on the velocity is presented in Figure 1. it is observed that there is a rise in the velocity due to enhancement of thermal buoyancy force. Figure 2 presents typical velocity profiles in the boundary layer for various values of the solutal Grashof number $G c$, while all other parameters are kept at some fixed values. As expected, the fluid velocity increases and the peak value is more distinctive due to increase in the species buoyancy force

For various values of the magnetic parameter $M$, the velocity profiles are plotted in Figure 3 . It can be seen that as $M$ increases, the velocity decreases. This result qualitatively agrees with the expectations, since the magnetic field exerts a retarding force on the free convection flow. The effect of the permeability parameter $K$ on the velocity field is shown in Figure 4 . An increase in $K$ will therefore increase the resistance of the porous medium (as the permeability physically 
become less with increasing $K^{\prime}$ ) which will tend to decelerate the flow and reduce the velocity. This behavior is evident from Figure 4.

Figures 5(a) and 5(b) illustrate the velocity and temperature profiles for different values of the Prandtl number Pr. The numerical results show that the effect of increasing values of Prandtl number results in a decreasing velocity (Fig.5 (a)). From Figure 5 (b), it is observed that an increase in the Prandtl number results a decrease of the thermal boundary thickness and in general lower average temperature with in the boundary layer.

The influence of the thermal radiation parameter $N$ on the velocity and temperature are shown in Figs. 6(a) and 6(b) respectively. It is obvious that an increase in the radiation parameter results in a decrease in the velocity and temperature within the boundary layer.

For different values of the heat generation parameter $Q$, the velocity and temperature profiles are plotted in Figs. 7 (a) and 7 (b) respectively. From Figure 7 (a), it is seen that due to the heat generation the buoyancy force increases which induces the flow rate to increase giving rise to an increase in the velocity profiles. Figure 7 (b) shows the variation of the temperature profiles for different values of $Q$. It is seen that, the temperature profiles increase with an increase in the heat generation parameter $Q$.

For different values of the Schimidt number $S c$, the velocity and concentration profiles are plotted in Figs. 8 (a) and 8(b) respectively. As the Schimidt number Sc increases the concentration decreases. The reductions in the velocity and concentration profiles are accompanied by simultaneous reductions in the velocity and concentration boundary layer, which is evident from Figs. 8 (a) and $8(\mathrm{~b})$.

Figures 9 (a) and $9(\mathrm{~b})$ show the velocity and concentration profiles for different values of chemical reaction parameter $K r$. it is observed that an increase in the chemical reaction parameter results in a decrease in the velocity and concentration.

The effects of various governing parameters on the skin-friction coefficient $C_{f}$, Nusselt number $\mathrm{Nu}$, and Sherwood number Sh are shown in Tables 1 and 2. From Table 1, it is noticed that as Gr or $G c$ increases, there is a rise in the skin-friction coefficient, Nusselt number and the Sherwood number. It is seen that, as $M$ or $K$ increases, there is a fall in the skin-friction coefficient, Nusselt number and the Sherwood number. From Table 2, it is observed that an increase in the Prandtl number Pr leads to a fall in the skin-friction coefficient and the Sherwood number, while a rise in the Nusselt number. It is observed that an increase in radiation parameter $R$ leads to a decrease in the skin-friction and Sherwood number, while a rise in the Nusselt number. It is noticed that an increase in heat generation parameter $Q$ leads to rise in the skin-friction and Sherwood number, while a decrease in the Nusselt number. It is found that as the Schmidt number Sc increases, there is a fall in the skin friction coefficient and Nusselt number, while a rise in the Sherwood number. It is found that as the chemical reaction parameter increases, there is a fall in the skin friction coefficient and Nusselt number, while a rise in the Sherwood number. 


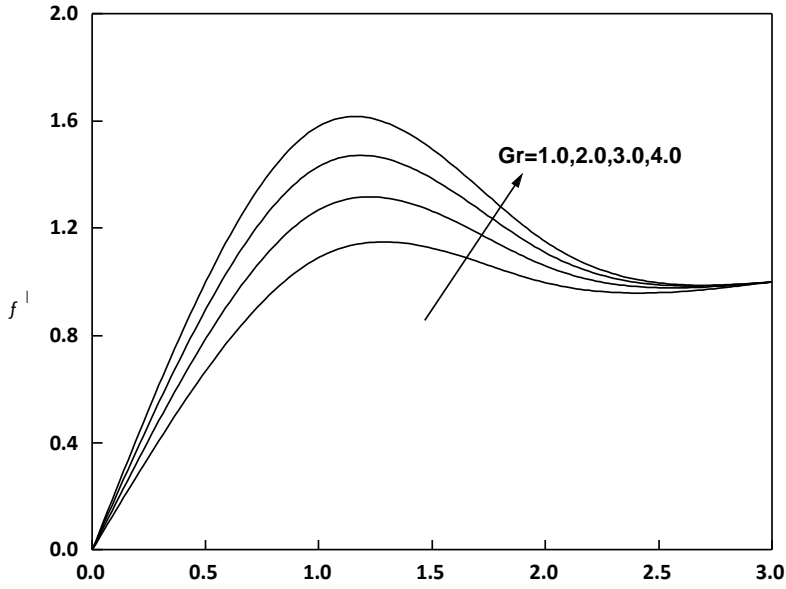

Fig. 1. Velocity profiles for different values of $G r$

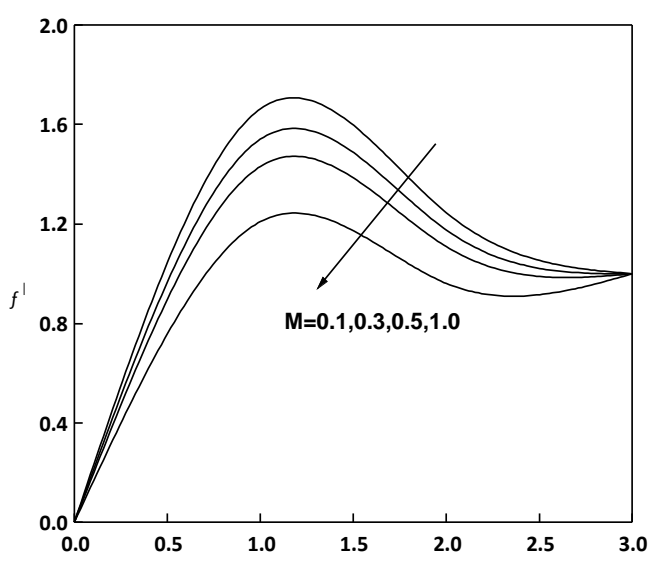

Fig. 3. Velocity profiles for different values of $M$

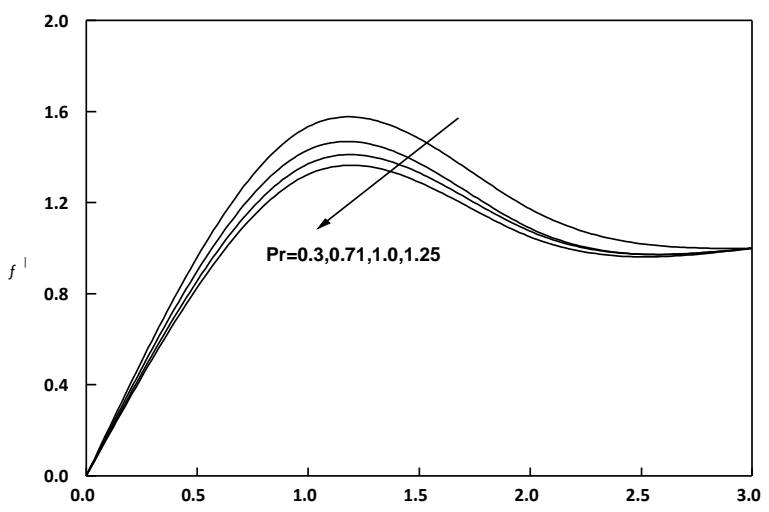

Fig. 5(a). Velocity profiles for different values of $\mathrm{Pr}$

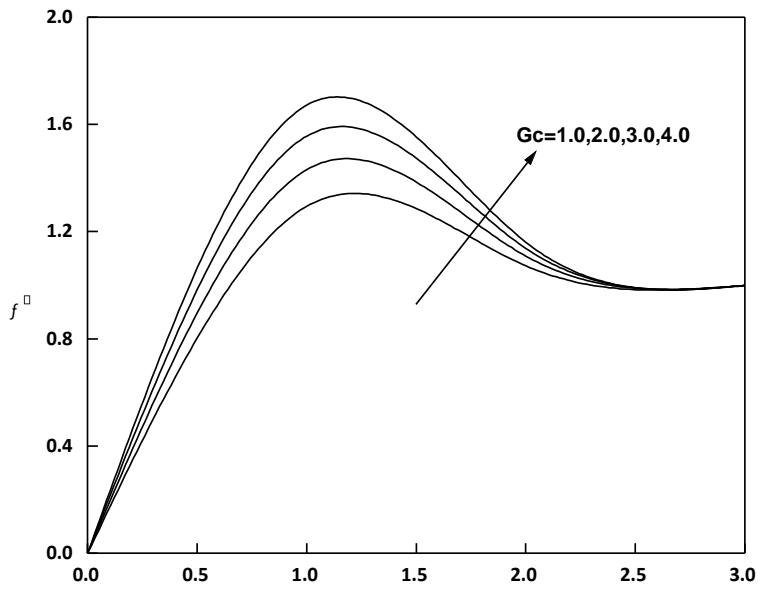

Fig. 2. velocity profiles for different values of Gc

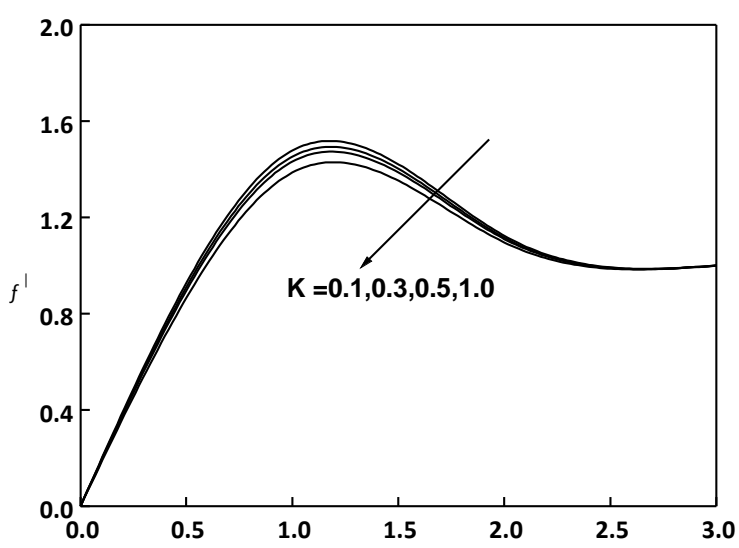

Fig. 4. Velocity profiles for different values of $K$

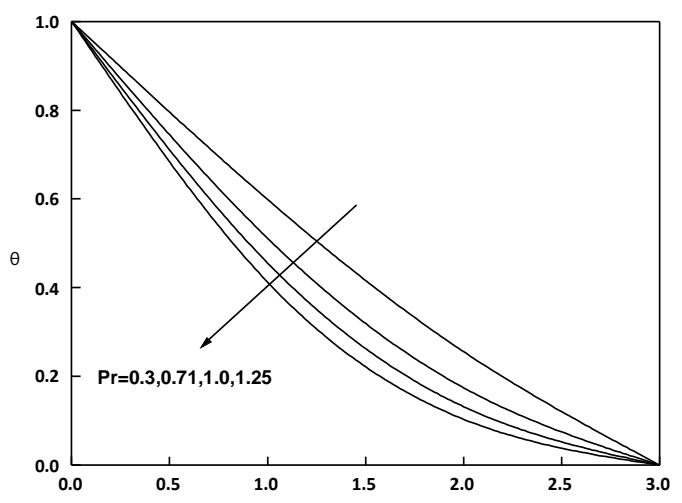

Fig. 5(b). Velocity profiles for different values of $\mathrm{Pr}$ 


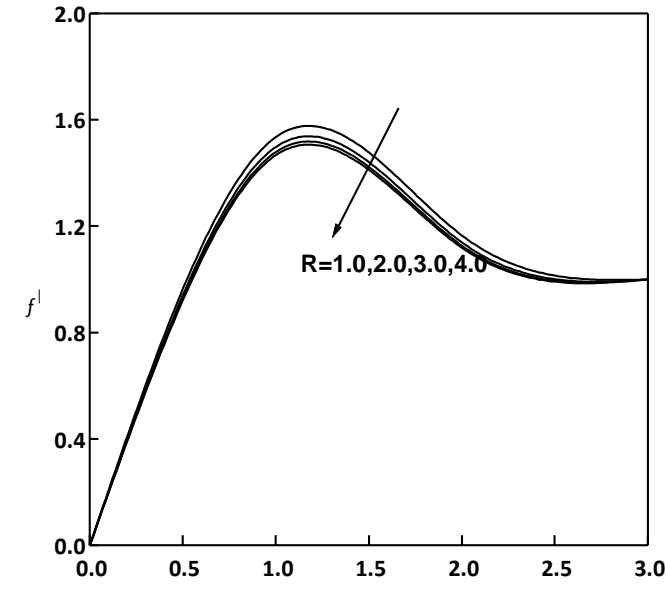

Fig. 6(a). Velocity profiles for different values of $R$

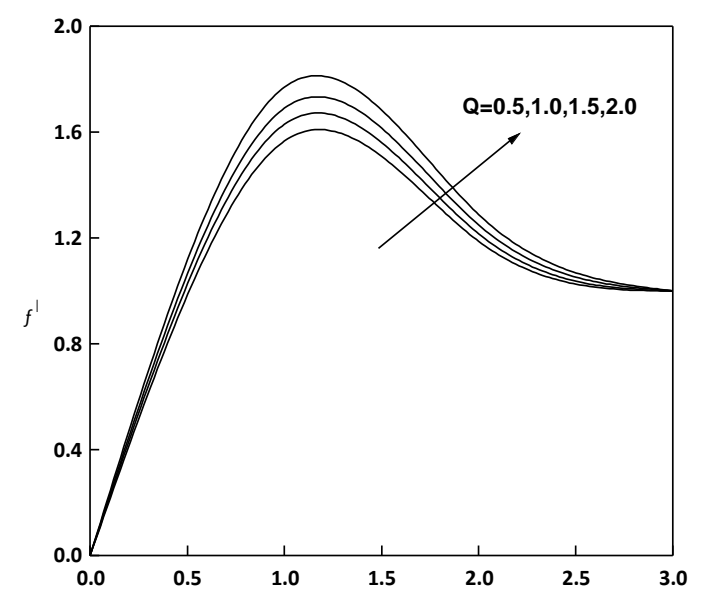

Fig. 7(a). Velocity profiles for different values of Q

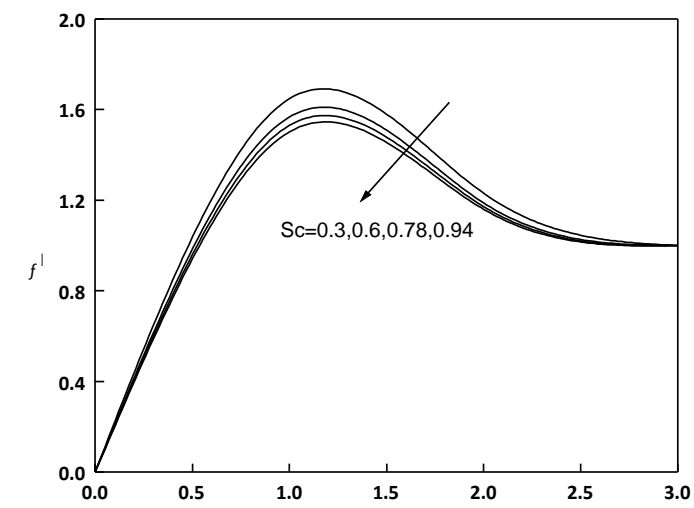

Fig. 8(a). Velocity profiles for different values of Sc

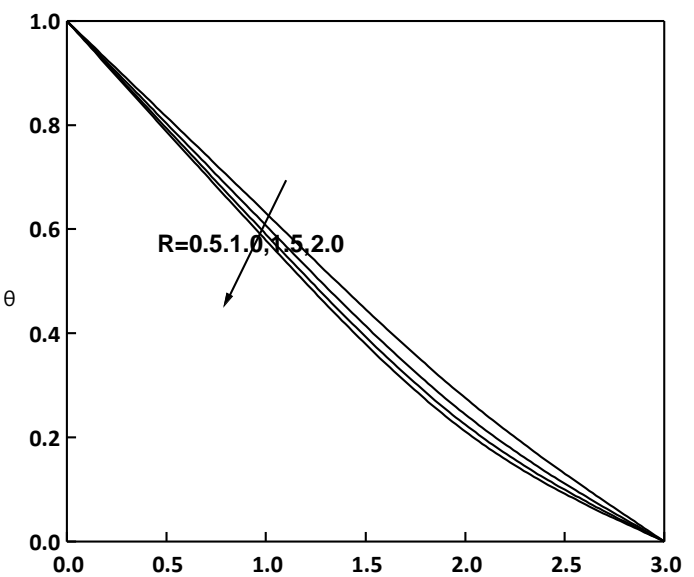

Fig. 6(b). Temperature profiles for different values of $R$

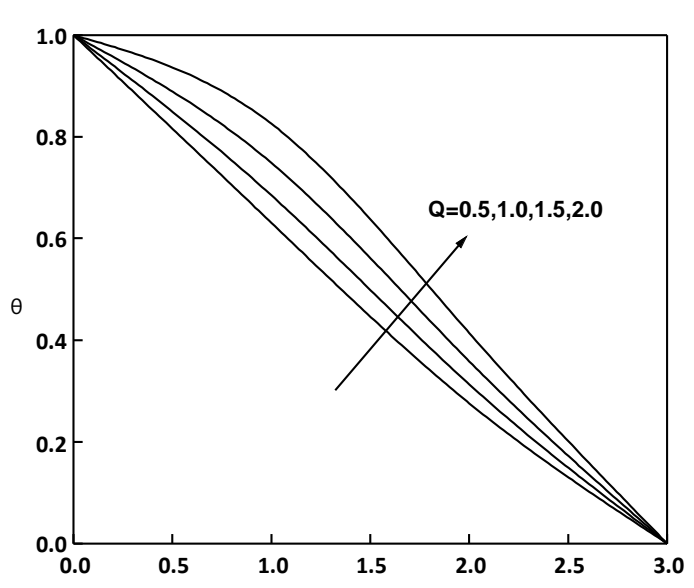

Fig. 7(b). Velocity profiles for different values of $Q$

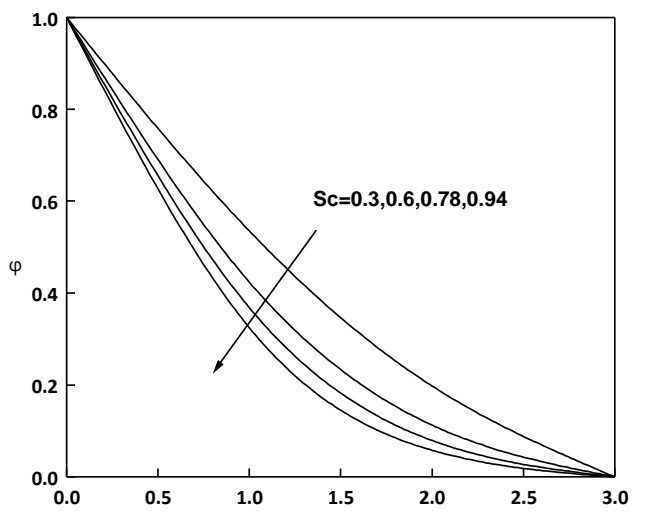

Fig. 8(b). Concentration profiles for different values of $S C$ 


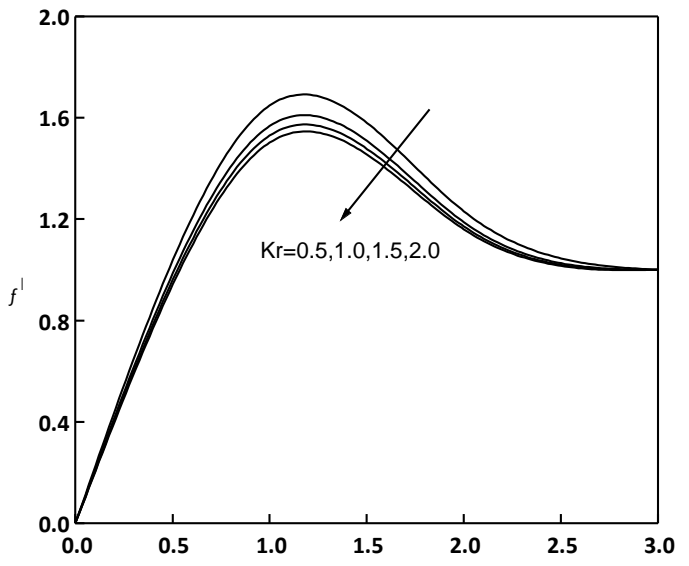

Fig. 9(a). Velocity profiles for different values of $K r$

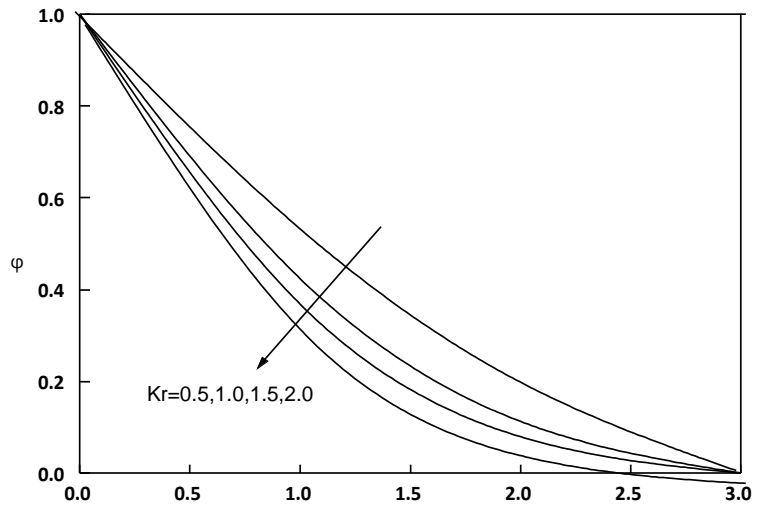

Fig. 9(b). Concentration profiles for values of $\mathrm{Kr}$

Table 1

Numerical values of the skin-friction coefficient $C_{f}$, Nusselt number $\mathrm{Nu}$ and Sherwood number $S h$ for $\operatorname{Pr}=0.71, R=0.5, Q=0.5, S c=0.6, S=0.5, K r=1.0$

\begin{tabular}{ccccccc}
\hline$G r$ & $G m$ & $M$ & $K$ & $C_{f}$ & $N u$ & $S h$ \\
\hline 2.0 & 2.0 & 0.5 & 0.5 & 3.4448 & 0.339924 & 0.665842 \\
4.0 & 2 & 0.5 & 0.5 & 4.77245 & 0.357057 & 0.71032 \\
2.0 & 4 & 0.5 & 0.5 & 4.51994 & 0.351674 & 0.697203 \\
2.0 & 2 & 1.0 & 0.5 & 3.03587 & 0.331013 & 0.643118 \\
2.0 & 2 & 0.5 & 1.0 & 3.34856 & 0.338677 & 0.662785 \\
\hline
\end{tabular}

Table 2

Numerical values of the skin-friction coefficient $C_{f}$, Nusselt number $\mathrm{Nu}$ and Sherwood number Sh for $G r=2.0, G m=2.0, M=0.5, K=0.5, S=1.0$

\begin{tabular}{crrrrrrr}
\hline$P r$ & $R$ & $Q$ & $S c$ & $K r$ & \multicolumn{1}{c}{$C_{f}$} & \multicolumn{1}{c}{$N u$} & \multicolumn{1}{c}{$S h$} \\
\hline 0.71 & 0.5 & 0.5 & 0.6 & 1.0 & 3.4448 & 0.339924 & 0.665842 \\
1.0 & 0.5 & 0.5 & 0.6 & 1.0 & 3.42564 & 0.3463 & 0.664573 \\
& & & & & & & \\
0.71 & 1.0 & 0.5 & 0.6 & 1.0 & 3.41767 & 0.349432 & 0.664051 \\
& & & & & & & \\
0.71 & 0.5 & 1.0 & 0.6 & 1.0 & 3.51712 & 0.235045 & 0.669766 \\
& & & & & & & \\
0.71 & 0.5 & 0.5 & 1.0 & 1.0 & 3.30131 & 0.216948 & 0.685779 \\
0.71 & 0.5 & 0.5 & 1.0 & 2.0 & 3.2654 & 0.19812 & 0.7569 \\
\hline
\end{tabular}




\section{Conclusions}

In this paper The effects of various governing parameters on the velocity, temperature, concentration, skin-friction coefficient, Nusselt number and Sherwood number are shown in figures and tables and analyzed in detail.

$>$ It is noticed that an increase in heat generation parameter $Q$ leads to rise in the skin-friction and Sherwood number, while a decrease in the Nusselt number.

$>$ It is found that as the Schmidt number Sc increases, there is a fall in the skinfriction coefficient and Nusselt number, while a rise in the Sherwood number.

$>$ It is found that as the chemical reaction parameter increases, there is a fall in the skinfriction coefficient and Nusselt number, while a rise in the Sherwood number.

\section{Acknowledgement}

Author is thankful to the referee for the valuable suggestions who help to improve the quality of this manuscript

\section{References}

[1] Lai, F. C., and F. A. Kulacki. "Non-Darcy mixed convection along a vertical wall in a saturated porous medium." Journal of Heat Transfer (Transcations of the ASME (American Society of Mechanical Engineers), Series C);(United States) 113, no. 1 (1991). https://doi.org/10.1115/1.2910537

[2] Hsu, C. T., and P. Cheng. "The Brinkman model for natural convection about a semi-infinite vertical flat plate in a porous medium." International Journal of Heat and Mass Transfer 28, no. 3 (1985): 683-697. https://doi.org/10.1016/0017-9310(85)90190-5

[3] Vafai, Kambiz, and Chang L. Tien. "Boundary and inertia effects on flow and heat transfer in porous media." International Journal of Heat and Mass Transfer 24, no. 2 (1981): 195-203. https://doi.org/10.1016/0017-9310(81)90027-2

[4] Bejan, Adrian, and Khairy R. Khair. "Heat and mass transfer by natural convection in a porous medium." International Journal of Heat and Mass Transfer 28, no. 5 (1985): 909-918. https://doi.org/10.1016/0017-9310(85)90272-8

[5] Raptis, A. A. "Flow through a porous medium in the presence of a magnetic field." International journal of energy research 10, no. 1 (1986): 97-100. https://doi.org/10.1002/er.4440100112

[6] Helmy, Kamal Anwar. "MHD unsteady free convection flow past a vertical porous plate." ZAMM-Journal of Applied Mathematics and Mechanics/Zeitschrift für Angewandte Mathematik und Mechanik: Applied Mathematics and Mechanics 78, no. 4 (1998): 255-270. https://doi.org/10.1002/(SICI)15214001(199804)78:4<255::AID-ZAMM255>3.0.CO;2-V

[7] Elbashbeshy, E. M. A. "Heat and mass transfer along a vertical plate with variable surface tension and concentration in the presence of the magnetic field." International Journal of Engineering Science 35, no. 5 (1997): 515-522. https://doi.org/10.1016/S0020-7225(96)00089-4

[8] Chamkha, Ali J., and Abdul-Rahim A. Khaled. "Similarity solutions for hydromagnetic simultaneous heat and mass transfer by natural convection from an inclined plate with internal heat generation or absorption." Heat and Mass Transfer 37, no. 2-3 (2001): 117-123. https://doi.org/10.1007/s002310000131

[9] Cess, R. D. "The interaction of thermal radiation with free convection heat transfer." International Journal of Heat and mass transfer 9, no. 11 (1966): 1269-1277. https://doi.org/10.1016/0017-9310(66)90119-0

[10] Arpaci, Vedat S. "Effect of thermal radiation on the laminar free convection from a heated vertical plate." International Journal of Heat and Mass Transfer 11, no. 5 (1968): 871-881. https://doi.org/10.1016/0017-9310(68)90130-0

[11] Raptis, A. "Radiation and free convection flow through a porous medium." International Communications in Heat and Mass Transfer 25, no. 2 (1998): 289-295. https://doi.org/10.1016/S0735-1933(98)00016-5

[12] Ibrahim, S. Mohammed, and N. Bhaskar Reddy. "Radiation and mass transfer effects on MHD free convection flow along a stretching surface with viscous dissipation and heat generation." International Journal of Applied Mathematics and Mechanics 8, no. 8 (2012): 1-21. 
[13] Damseh, Rebhi A., H. M. Duwairi, and M. Al-Odat. "Similarity analysis of magnetic field and thermal radiation effects on forced convection flow." Turkish Journal of Engineering and Environmental Sciences 30, no. 2 (2006): 83-89.

[14] Vajravelu, K., and A. Hadjinicolaou. "Heat transfer in a viscous fluid over a stretching sheet with viscous dissipation and internal heat generation." International Communications in Heat and Mass Transfer 20, no. 3 (1993): 417-430. https://doi.org/10.1016/0735-1933(93)90026-R

[15] Hossain, M. A., M. M. Molla, and L. S. Yaa. "Natural convection flow along a vertical wavy surface temperature in the presence of heat generation/absorption." Int. J. Thermal Science 43 (2004): 157-163. https://doi.org/10.1016/j.ijthermalsci.2003.04.001

[16] Lavanya, Bommanna. "MHD Rotating Flow Through a Porous Medium with Heat and Mass Transfer." Journal of Advanced Research in Fluid Mechanics and Thermal Sciences 54, no. 2 (2019): 221-231.

[17] Nagasasikala, Madduleti, and Bommanna Lavanya. "Effects of Dissipation and Radiation on Heat Transfer Flow of a Convective Rotating Cuo-Water Nano-fluid in a Vertical Channel." Journal of Advanced Research in Fluid Mechanics and Thermal Sciences 50, no. 2 (2018): 108-117.

[18] Nagasasikala, Madduleti, and G. Phrabhakar Rao. "Heat and mass transfer of a MHD flow of a nanofluid through a porous medium in an annular, circular region with outer cylinder maintained at constant heat flux." CFD Letters 11, no. 9 (2019): 32-58.

[19] Hamisu, Muhammad Tukur, Mahmud Muhammad Jamil, Umar Sanusi Umar, and Aisha Sa'ad. "Numerical Study Of Flow In Asymmetric 2D Plane Diffusers With Different Inlet Channel Lengths." CFD Letters 11, no. 5 (2019): 1-21

[20] Lee, Y. K. "The use of nanofluids in domestic water heat exchanger." J. Adv. Res. Appl. Mech 3, no. 1 (2014): 9-24. 